\title{
RECONHECIMENTO DE SENTENÇAS NO SILÊNCIO E NO RUÍDO VERSUS BENEFÍCIO DE CRIANÇAS E ADOLESCENTES USUÁRIOS DE PRÓTESES AUDITIVAS
}

\author{
Recognition of Portuguese sentences lists test in silence and noise \\ versus benefits for children and teenagers using hearing aids \\ Enma Mariángel Ortiz Torres ${ }^{(1)}$, Alexandre Hundertmarck Lessa ${ }^{(2)}$, \\ Nilvia Herondina Soares Aurélio ${ }^{(3)}$, Sinéia Neujahr dos Santos ${ }^{(4)}$, Maristela Júlio Costa ${ }^{(5)}$
}

\section{RESUMO}

Objetivo: avaliar crianças e adolescentes, usuários de próteses auditivas, buscando investigar o benefício proporcionado pela amplificação sonora por meio do teste Listas de Sentenças em Português e do Questionário de avaliação do benefício em crianças e adolescentes, e verificar se existe correlação entre os resultados obtidos por meio dos dois instrumentos de avaliação. Método: estudo retrospectivo, onde foram avaliados 13 sujeitos com idades entre oito e 14 anos, de ambos os sexos, com perda auditiva de no máximo, grau moderadamente severo na melhor orelha, usuários de próteses auditivas bilateralmente há mais de dez meses e protetizados por um Programa de Saúde Auditiva. Foi realizada anamnese, nova avaliação audiológica básica e aplicados o teste Listas de Sentenças em Português e o Questionário de Avaliação do Beneficio em crianças e adolescentes. Resultados: os resultados obtidos no Teste de Listas de Sentenças em Português e no questionário mostraram que houve benefício com o uso das próteses, tanto no silêncio, quanto no ruído. Entretanto, não houve correlação estatisticamente significante entre os resultados. Conclusão: embora não tenha sido verificada correlação estatisticamente significante entre os resultados obtidos por meio dos dois instrumentos de avaliação, foi observada melhora no desempenho de reconhecimento de fala fazendo uso das próteses através do teste Listas de Sentenças em Português, tanto na avaliação no ruído, quanto no silêncio e, em resposta ao questionário, os avaliados referiram benefício com o uso da amplificação sonora.

DESCRITORES: Criança; Perda Auditiva; Auxiliares de Audição; Discriminação da Fala; Questionários

\section{INTRODUÇÃO}

A audição é o sentido que promove a comunicação humana ${ }^{1}$. Os processos da linguagem e

(1) Fonoaudióloga; Mestranda em Distúrbios da Comunicação Humana pela Universidade Federal de Santa Maria.

(2) Fonoaudiólogo; Mestrando em Distúrbios da Comunicação Humana pela Universidade Federal de Santa Maria.

(3) Fonoaudióloga; Mestre em Distúrbios da Comunicação Humana pela Universidade Federal de Santa Maria.

(4) Fonoaudióloga; Doutoranda em Distúrbios da Comunicação Humana pela Universidade Federal de Santa Maria.

(5) Fonoaudióloga; Professora Adjunta do Departamento de Fonoaudiologia da Universidade Federal de Santa Maria; Doutora em Ciências dos Distúrbios da Comunicação Humana pela Universidade Federal de São Paulo.

Conflito de interesses: inexistente da aprendizagem são bastante complexos, envolvendo redes de neurônios distribuídas em diferentes regiões cerebrais e se relacionam à percepção da fala, sendo dependentes da integridade auditiva periférica e central².

A perda auditiva é definida como uma diminuição da audição em qualquer grau que reduza a inteligibilidade da mensagem falada para a interpretação apurada ou para a aprendizagem ${ }^{3}$.

Quando manifestada na infância, a perda auditiva, mesmo leve, pode originar dificuldades escolares, problemas de desenvolvimento de linguagem e distúrbios comportamentais. A perda auditiva na infância é um importante problema de saúde pública, tanto pela freqüência como pelos intensos 
prejuízos lingüísticos, educacionais e psicossociais que pode determinar ${ }^{3}$.

Para minimizar os efeitos negativos da deficiência auditiva, foram desenvolvidas as próteses auditivas, as quais são consideradas o componente fundamental da reabilitação auditiva. A amplificação sonora proporcionada pelas próteses auditivas não se restringe aos sons da fala, mas engloba sons ambientais, sinais de perigo e de alerta e outros sons presentes na vida do usuário desse aparato, além de facilitar a educação e o desenvolvimento psicossocial e intelectual do sujeito com perda auditiva ${ }^{4}$.

O uso da prótese auditiva representa uma grande chance de se modificar os rumos da relação da criança deficiente auditiva com o meio em que vive, mas para ser considerada bem adaptada, a prótese deve propiciar benefícios ao usuário ${ }^{5}$. Portanto, cabe ao fonoaudiólogo, avaliar os benefícios e as limitações das habilidades de escuta da criança ou jovem utilizando a prótese auditiva, ou seja, quais os efeitos da amplificação sonora na qualidade de vida destes indivíduos ${ }^{6}$.

Para verificar os benefícios da amplificação sonora na qualidade de vida de crianças e jovens, o fonoaudiólogo pode utilizar procedimentos como, observar os comportamentos da criança em resposta ao sinal amplificado fornecido pela prótese auditiva, quantificar as respostas comportamentais da criança utilizando a amplificação por meio do ganho funcional e de testes de percepção da fala (medidas diretas); como também, analisar as medidas indiretas do desempenho da criança obtidas em entrevistas com pais, cuidadores e/ou educadores por meio de questionários desenvolvidos para tal finalidade ${ }^{6}$.

Os testes de reconhecimento de fala mais aplicados, geralmente, utilizam como estímulo palavras isoladas, como o teste Lista de Monossílabos para discriminação vocal ${ }^{7}$, utilizado no Brasil, e os testes PAL PB ${ }^{8}$ e CID W $22^{9}$, utilizados nos Estados Unidos $^{10}$. No entanto, testes de reconhecimento de fala com palavras isoladas não atendem a necessidade de avaliar o indivíduo em uma situação que se assemelhe àquela vivida no dia-a-dia, pois fornecem informações fragmentadas do funcionamento do sistema auditivo ${ }^{11}$. Por outro lado, os testes que utilizam sentenças como estímulo permitem avaliar o comportamento auditivo do indivíduo em situações mais próximas as do seu dia a dia.

Por constituírem o melhor instrumento para avaliar a comunicação dos indivíduos em suas atividades diárias, com ou sem prótese auditiva, surgiram testes de fala que utilizam como estímulo sentenças de uso frequente. $O$ desempenho do indivíduo no reconhecimento destas frases nos dá uma medida direta de como ele é capaz de participar de uma conversação ${ }^{12}$. A partir dessas considerações, foi desenvolvido o teste Listas de Sentenças em Português (LSP) ${ }^{13}$, que avalia o reconhecimento da fala com sentenças, no silêncio e no ruído, e nos fornece informações sobre a real capacidade de comunicação do paciente.

Os testes de reconhecimento de fala são, usualmente, utilizados para avaliação de adultos, porém, em crianças e adolescentes estes testes que avaliam o reconhecimento de fala no silêncio e no ruído, podem revelar dificuldades perceptuais auditivas, assim como, evidenciar melhoras advindas do uso da amplificação. Considerando esses aspectos, percebe-se a necessidade de avaliar esta população com um material sensível, que nos forneça também dados qualitativos sobre a capacidade de comunicação do paciente.

A avaliação auditiva em crianças e adolescentes, utilizando sentenças como estímulo, tanto no silêncio, como no ruído, ainda não faz parte da rotina audiológica utilizada no Brasil. Porém, existem na literatura pesquisas ${ }^{14,15}$, onde crianças foram avaliadas utilizando as sentenças do LSP como estímulo. No entanto, utilizar tal teste em crianças e adolescentes com perda auditiva, para pesquisar o benefício advindo do uso da amplificação sonora, é um trabalho inovador, já que não foram encontrados trabalhos semelhantes na literatura nacional.

Para investigar a percepção do paciente e da família sobre as dificuldades de comunicação, monitorar seu progresso e localizar suas necessidades auditivas fora da bateria audiométrica padrão, o fonoaudiólogo também pode utilizar as escalas e questionários de auto-avaliação, os quais nos fornecem medidas quantificáveis do benefício percebido e atuam como preditores das dificuldades enfrentadas pelos usuários de próteses auditivas, auxiliando no ajuste das mesmas ${ }^{16,17}$.

Com base nestes propósitos, os objetivos desta pesquisa foram investigar o benefício proporcionado pela amplificação sonora por meio do teste Listas de Sentenças em Português e do Questionário de avaliação do benefício em crianças e adolescentes, usuários de próteses auditivas e verificar se existe correlação entre os resultados obtidos por meio dos dois instrumentos de avaliação.

\section{MÉTODO}

Este estudo retrospectivo foi realizado no Laboratório de Próteses Auditivas do Serviço de Atendimento Fonoaudiológico (SAF) de uma instituição de ensino superior, no período compreendido entre maio e agosto de 2009. 
Os pacientes estudados foram protetizados pelo programa de concessão de próteses auditivas de fluxo contínuo da Secretaria de Assistência à Saúde do Ministério da Saúde, mediante credenciamento celebrado entre a Secretaria de Saúde do Estado do Rio Grande do Sul e a instituição de ensino superior, a partir de 2005, com base nas Portarias 587 e 589, da Secretaria de Assistência à Saúde do Ministério da Saúde, publicadas em outubro de 2004 e desenvolvido na referida instituição.

Para a seleção dos indivíduos, foram utilizados os seguintes critérios de inclusão: terem idade entre oito e 14 anos; apresentarem perda auditiva de no máximo, grau moderadamente severo na melhor orelha (56 a $70 \mathrm{~dB} N A)^{18}$, para que fosse possível a resposta ao teste de reconhecimento de sentenças; terem adaptado próteses auditivas retroauriculares, de forma binaural, de tecnologia $A, B, C$ segundo classificação sugerida pelo Ministério da Saúde, há no mínimo, seis meses no Laboratório de Próteses Auditivas, por meio do programa de concessão de próteses auditivas, da referida instituição de ensino superior, credenciada pela Secretaria de Assistência à Saúde do Ministério da Saúde, conforme citado anteriormente.

Os critérios de exclusão adotados foram: presença de alterações neurológicas, presença de rolha de cerúmen ou de outras alterações no meato acústico externo capazes de alterar o desempenho no teste, ou de orelha média, nos casos que apresentassem curvas timpanométricas tipo $B$, incapacidade de responder ao teste ou dificuldade para memorizar as sentenças do teste Listas de Sentenças em Português ${ }^{7}$

Participaram deste estudo somente indivíduos cujos pais ou responsáveis concordaram com a realização dos procedimentos necessários para a execução da pesquisa e assinaram o Termo de Consentimento Livre e Esclarecido, após terem recebido esclarecimentos sobre o objetivo e metodologia do estudo proposto.

Com base nestes critérios, foram selecionados 70 pacientes para serem contatados e convidados a participar da pesquisa e realizar as avaliações.

Antes da realização das avaliações, foi realizada a meatoscopia, e aplicada uma anamnese ao paciente e/ou seu familiar ou responsável, com o intuito de pesquisar aspectos referentes aos dados pessoais; escolaridade do paciente e frequência do uso das próteses auditivas. A seguir foram realizadas as medidas de imitanciometria e pesquisa dos reflexos estapedianos.

$\mathrm{Na}$ sequência, foram verificadas as condições técnicas das próteses auditivas, tendo sido avaliados apenas os indivíduos cujas próteses estavam em boas condições de funcionamento, conferidas com base nas informações dadas pelo paciente ou familiar, além da escuta das próteses auditivas pelo avaliador, a seguir das medidas de ganho funcional, e avaliação de possíveis distorções verificadas com o auxílio do equipamento de verificação das próteses auditivas.

Após estes procedimentos, aqueles pacientes que não possuíam cerúmen ou qualquer alteração de orelha externa ou média que pudesse interferir no teste, ou ainda problemas técnicos nas próteses, foram submetidos à pesquisa dos Limiares de Reconhecimento de Sentenças no Silêncio e no Ruído (LRSS e LRSR) e dos Índices Percentuais de Reconhecimento de Sentenças no Silêncio e no Ruído (IPRSS e IPRSR) ${ }^{13}$, em campo livre. Estes medidas foram realizadas na mesma sessão de avaliação, porém o paciente foi avaliado sem o uso das próteses auditivas (LRSS e LRSR, IPRSS e IPRSR) e a seguir, fazendo uso das mesmas (IPRSS e IPRSR).

Alguns indivíduos não foram capazes de responder ao teste de reconhecimento de sentenças, tendo sido também excluídos, e assim, o grupo estudado ficou composto por 13 sujeitos, sendo quatro do sexo feminino e nove do sexo masculino; usuários de protetização bilateral, há mais de dez meses; com idades variando de oito a 14 anos e média de 12,3 anos os quais foram capazes de realizar todas as avaliações .

Para a obtenção dos LRSS, IPRSS, LRSR e o IPRSR, foi utilizado o teste Listas de Sentenças em Português ${ }^{13}$, o qual é constituído por uma lista de 25 sentenças, outras sete listas com 10 sentenças e um ruído com espectro de fala. As sentenças e o ruído estão gravados em $C D$, em canais independentes.

Para obtenção das medidas de imitanciometria, foi utilizado o Imitanciometro AZ, marca Interacoustics, e para as demais avaliações, foi utilizado um audiômetro digital de dois canais, marca Damplex, modelo DA65 e um sistema de amplificação para audiometria em campo livre, modelo TA 1010. As sentenças foram apresentadas utilizando-se um CD Player da marca Britânia, modelo B5279, na opção lineout acoplada ao audiômetro.

O procedimento utilizado para a aplicação do teste foi baseado na estratégia denominada sequencial ou adaptativa ou ainda, ascendente-descendente ${ }^{19}$. Esta permite determinar o limiar de reconhecimento de fala, nível necessário para o indivíduo identificar de forma correta aproximadamente $50 \%$ dos estímulos apresentados.

Inicialmente foi realizado um treinamento para que os sujeitos se familiarizassem com o teste e fossem obtidos os limiares aproximados sem o uso das próteses auditivas. As dez primeiras sentenças 
da lista $1 \mathrm{~A}$ foram aplicadas aos indivíduos antes da obtenção dos LRSS e dos LRSR.

O procedimento da pesquisa dos LRSS e LRSR, sem a utilização das próteses auditivas, consistiu na apresentação de um estímulo em uma determinada intensidade, obtida após o treino para a realização do teste. Se o indivíduo foi capaz de reconhecer corretamente o estímulo de fala apresentado, a intensidade do mesmo foi diminuída em intervalos preestabelecidos. Caso contrário, sua intensidade foi aumentada. Este procedimento foi repetido até o final da lista. O LRSR foi pesquisado com uma intensidade de ruído constante, a 65 dB NPS (A). Foram utilizados intervalos de $4 \mathrm{~dB}$ até a primeira mudança no tipo de resposta e, posteriormente, os intervalos de apresentação dos estímulos passaram a $2 \mathrm{~dB}$ entre si até o final da lista ${ }^{19}$.

É importante salientar que o LRSS e LRSR não foram analisados neste estudo, eles foram obtidos com os pacientes sem a próteses auditivas, apenas para servirem de base para estabelecer a intensidade na qual deveriam ser pesquisados os IPRSS e IPRSR, com e sem as próteses auditivas,.

Assim sendo, na pesquisa dos IPRSS e IPRSR, a intensidade de apresentação das sentenças foi estabelecida com base nos LRSS e LRSR respectivamente, de cada individuo, ou seja, para a pesquisa do IPRSS, a fala foi fixada na intensidade, na qual foi obtido o LRSS e então apresentadas todas as sentenças de uma lista. Posteriormente, foi calculada a porcentagem de acertos por lista nesta condição. Enquanto que, para a medida do IPRSR, foi fixada a relação S/R na qual foi obtido o LRSR e então apresentadas as sentenças de cada, para que também fosse calculada a porcentagem de acertos obtidos em uma relação $S / R$ fixa. Nas pesquisas no ruído, este permaneceu constante a 65 dB NPS (A).

Esta estratégia foi usada para a obtenção dos IPRSS e IPRSR sem o uso das próteses, e a seguir, com o uso das próteses, e então calculada a diferença entre a porcentagem de acertos obtida nas duas condições, o que serviu para dimensionar o benefício no que se referiu ao reconhecimento de fala com o uso das próteses auditivas.

As medidas em campo livre foram pesquisadas após a devida calibração do equipamento, por um profissional registrado no Inmetro SP, a fim de estabelecer os níveis de pressão sonora da fala e ruído, tendo em vista as características do sinal de teste e as condições acústicas do ambiente. Para isso, foi utilizado Microfone B\&K $4144 \mathrm{n} / \mathrm{s}$, Medidor de Nível de Pressão Sonora B\&K 2260 n/s e Calibrador de Nível de Pressão Sonora B\&K n/s. O medidor foi posicionado em um ponto médio entre as duas orelhas, a uma distância de um metro do alto-falante.

O VU-meter do audiômetro foi colocado na posição 0 antes de cada avaliação e cada canal do $C D$ foi calibrado separadamente, sendo que um tom puro de $1000 \mathrm{~Hz}$ foi utilizado para calibrar o canal das sentenças. O uso do tom puro é necessário, pois a fala é um som complexo que apresenta uma variação de $30 \mathrm{~dB}$ entre o som mais intenso e o menos intenso, oscilando $12 \mathrm{~dB}$ acima e 18 $\mathrm{dB}$ abaixo da média ${ }^{20}$. Portanto, o uso de um som contínuo de referência mantém sempre as mesmas condições de apresentação. Já o canal do ruído foi calibrado usando o próprio ruído gravado no outro canal.

Foi usado um medidor digital do nível de pressão sonora (NPS), marca Radio Schack, periodicamente, para monitorar os níveis de apresentação dos diferentes estímulos, determinando e garantindo sempre as mesmas condições acústicas no campo livre para todos os sujeitos avaliados.

E finalmente, o benefício proporcionado pela amplificação, nos pacientes com idade entre oito e 14 anos, foi pesquisado por meio do Questionário de Avaliação do Beneficio do AASI em Crianças e Adolescentes ${ }^{21}$. Este instrumento contém questões fechadas, as quais são acompanhadas de gravuras ilustrativas referentes ao benefício proporcionado pelas próteses auditivas no ambiente residencial, na escola e no convívio social. Este questionário foi respondido pelo próprio paciente.

O questionário possui 11 questões, às quais foram atribuídas pontos. Cada uma das respostas fechadas de cada item, foram pontuadas com ' 2 ' (para a resposta 'sim'), '1' (para a resposta 'um pouco') e '0' (para a resposta 'não)', de maneira que a pontuação máxima para o questionário é de 22 pontos e a mínima de zero, o que denota que quanto maior a pontuação no teste, maior o benefício percebido pelo usuário.

As questões identificadas pela letra " $A$ " referem-se ao ambiente "casa", já as questões "B" correspondem ao ambiente "escola" e, por último, as questões " $C$ " correspondem ao ambiente "social".

Esta pesquisa é um subprojeto dentro de um projeto maior intitulado: Pesquisa e Base de Dados em Saúde Auditiva, registrado no Gabinete de Projetos sob o no 019731 e aprovado pelo Comitê de Ética em Pesquisa com certificado de no 0138.0.243.246-06, em 05/12/2006.

O teste SKtest foi utilizado para verificar a distribuição da amostra. O teste de T Pareado foi aplicado para análise da significância no desempenho dos participantes nas pesquisas do IPRSS e IPRSR com e sem o uso das próteses auditivas. O nível de rejeição da hipótese nula foi fixado em um valor 
menor ou igual a $5 \%$. Nas tabelas, os resultados estatísticos foram assinalados com um asterisco $\left({ }^{*}\right)$ quando significantes.

O teste Coeficiente de Correlação de Pearson foi utilizado para verificar a existência de correlação entre os IPRSS e IPRSR, com e sem o uso das próteses em ambos os casos, com a pontuação média obtida no questionário.

\section{RESULTADOS}

Serão apresentados nas tabelas: os dados referentes ao tempo de uso das próteses auditivas, os resultados das medidas obtidas com o teste de reconhecimento de fala (LSP) e os resultados obtidos a partir do Questionário de Avaliação do Benefício do AASI em Crianças e Jovens.

Tabela 1 - Tempo de uso diário das próteses auditivas

\begin{tabular}{ccc}
\hline Tempo de uso diário das próteses auditivas & $\%$ & $\mathbf{N}=\mathbf{1 3}$ \\
\hline $\mathbf{0}$ a $\mathbf{4}$ horas & 7,70 & 01 \\
$\mathbf{5}$ a $\mathbf{8}$ horas & 46,20 & 06 \\
$\mathbf{9}$ a $\mathbf{1 2}$ horas & 30,80 & 04 \\
mais de 12 horas & 15,40 & 02 \\
\hline
\end{tabular}

\section{Resultados obtidos com o teste LSP}

Na tabela 2 encontram-se os valores das médias e medianas dos IPRSS e IPRSR, com e sem o uso das próteses auditivas, assim como os valores mínimos e máximos apresentados nas pesquisas dos mesmos. O teste SKtest mostrou que os dados apresentam distribuição normal.

Tabela 2 - Valores das médias, medianas, desvios padrão, limites mínimos e máximos dos IPRSS e IPRSR, apresentados em valores percentuais e Teste de Normalidade

\begin{tabular}{cccccccc}
\hline N=13 & $\begin{array}{c}\text { Média } \\
\%\end{array}$ & $\begin{array}{c}\text { Mediana } \\
\%\end{array}$ & $\begin{array}{c}\text { DP } \\
\%\end{array}$ & $\begin{array}{c}\text { Mín } \\
\%\end{array}$ & $\begin{array}{c}\text { Max } \\
\%\end{array}$ & Sktest & \multirow{2}{*}{ Valor de p } \\
\hline IPRSSs & 63.08 & 70 & 17.50 & 40 & 90 & 0.1926 & \multirow{2}{*}{$0.0023^{*}$} \\
IPRSSc & 83.08 & 90 & 16.01 & 60 & 100 & 0.1793 & \\
\hline IPRSRs & 41.54 & 40 & 12.14 & 20 & 60 & 0.4891 & \multirow{2}{*}{$0.0001^{*}$} \\
IPRSRc & 69.23 & 60 & 19.35 & 40 & 100 & 0.5454 & \\
\hline
\end{tabular}

* valor estatisticamente significante $p \geq 0,05$

Legenda: IPRSSs: índice percentual de reconhecimento de sentenças no silêncio sem as próteses auditivas. IPRSSc: índice percentual de reconhecimento de sentenças no silêncio com as próteses auditivas. IPRSRs: índice percentual de reconhecimento de sentenças no ruído sem as próteses auditivas. IPRSRc: índice percentual de reconhecimento de sentenças no ruído com as próteses auditivas.

\section{Resultados obtidos com o Questionário de Avaliação do Benefício do AASI em Crianças e Jovens}

A tabela 3 mostra as pontuações obtidas em cada uma das perguntas do questionário, assim como a pontuação total por indivíduo e a pontuação total por pergunta.
Correlação entre os resultados do teste LSP e do Questionário de Avaliação do Benefício do AASI em Crianças e Jovens

A tabela 4 mostra o resultado da média dos pontos obtidos no questionário relacionando-os com os IPRSS e IPRSR com e sem o uso das próteses. Mostra também a correlação entre as diferenças dos resultados obtidos com e sem o uso das próteses, tanto na situação de silêncio, quanto na de ruído. Em todos os casos não foi observada correlação estatisticamente significante. 
Tabela 3 - Pontuação obtida no Questionário de Avaliação do Benefício do AASI em Crianças e Jovens

\begin{tabular}{cccccccccccc}
\hline A 1 & A 2 & A 3 & B 1 & B 2 & B 3 & B 4 & C 1 & C 2 & C 3 & C 4 & Pont ind \\
\hline 2 & 2 & 2 & $\#$ & $\#$ & $\#$ & $\#$ & 2 & 2 & 2 & 2 & 14 \\
2 & 2 & 2 & 2 & 2 & 2 & 2 & 2 & 2 & 2 & 1 & 21 \\
2 & 2 & 2 & 2 & 2 & 2 & 2 & 2 & 2 & 2 & 2 & 22 \\
2 & 1 & 2 & 2 & 1 & 2 & 1 & 1 & 1 & 2 & 0 & 15 \\
2 & 2 & 2 & 2 & 2 & 2 & 2 & 2 & 2 & 2 & 1 & 21 \\
2 & 2 & 2 & 2 & 2 & 2 & 1 & $\#$ & 2 & 2 & 1 & 18 \\
1 & 1 & 1 & 2 & 2 & 2 & 2 & $\#$ & $\#$ & 1 & 1 & 13 \\
2 & 2 & 2 & 2 & 2 & 2 & 2 & 2 & 2 & 2 & 0 & 20 \\
2 & 2 & 2 & 2 & 2 & 2 & 2 & 2 & 2 & 2 & 2 & 22 \\
2 & 2 & 2 & 1 & 2 & 2 & 2 & 2 & 2 & 2 & 2 & 21 \\
2 & 2 & 2 & 2 & 2 & 2 & 2 & 2 & 2 & 2 & 2 & 22 \\
2 & 2 & 2 & 2 & 2 & 2 & 2 & 2 & 2 & 2 & 2 & 22 \\
2 & 1 & 2 & 2 & 2 & 2 & 1 & 2 & 2 & 1 & 2 & 19 \\
\hline $\mathbf{2 5}$ & $\mathbf{2 3}$ & $\mathbf{2 5}$ & $\mathbf{2 3}$ & $\mathbf{2 3}$ & $\mathbf{2 4}$ & $\mathbf{2 1}$ & $\mathbf{2 1}$ & $\mathbf{2 3}$ & $\mathbf{2 4}$ & $\mathbf{1 8}$ & $\mathbf{1 9 , 2 3}$ \\
\hline
\end{tabular}

\# Sem resposta

Tabela 4 - Correlação entre IPRSS e IPRSR (com/ sem prótese e diferença) e pontuação média do questionário

\begin{tabular}{cc}
\hline & Correlação de Pearson \\
\hline IPRSSs & -0.1163 \\
IPRSSc & 0.2739 \\
IPRSSdif & 0.3085 \\
IPRSRs & -0.0944 \\
IPRSRc & 0.2287 \\
IPRSRdif & 0.2967 \\
\hline
\end{tabular}

* valor estatisticamente significante $p \geq 0,05$

Legenda: IPRSSs: índice percentual de reconhecimento de sentenças no silêncio sem as próteses auditivas. IPRSSc: índice percentual de reconhecimento de sentenças no silêncio com as próteses auditivas. IPRSRs: índice percentual de reconhecimento de sentenças no ruído sem as próteses auditivas. IPRSRc: índice percentual de reconhecimento de sentenças no ruído com as próteses auditivas.
A tabela 5 mostra os resultados das correlações entre a média dos pontos obtidos em cada uma das questões do questionário com o IPRSS e o IPRSR com e sem o uso das próteses, assim como a correlação entre as diferenças dos resultados obtidos com e sem o uso das próteses, tanto na situação de silêncio, quanto na de ruído. Os resultados mostram correlações aleatórias.

\section{DISCUSSÃO}

A partir dos resultados obtidos, serão discutidas questões a eles relacionadas.

Em relação ao tempo diário de uso das próteses auditivas, um significante número de sujeitos $(46,2 \%)$ referiu fazer uso das mesmas por cinco a oito horas diárias, o que constitui mais de um turno diariamente.

Tabela 5 - Correlação do IPRSS e IPRSR (com/sem prótese e a diferença) X pontuação por questão

\begin{tabular}{cccccccccccc}
\hline Corr & A1 & A2 & A3 & B1 & B2 & B3 & B4 & C1 & C2 & C3 & C4 \\
\hline IPRSSs & 0.2245 & 0.2088 & 0.2245 & -0.2445 & 0.3239 & -0.4621 & -0.4729 & $0.0334^{*}$ & 0.3117 & 0.0780 & 0.0906 \\
\hline IPRSSc & 0.4330 & 0.4656 & 0.4330 & -0.4410 & -0.0935 & -0.3175 & -0.1169 & 0.5109 & 0.6013 & 0.2239 & 0.2346 \\
\hline IPRSSdif & 0.1443 & 0.1826 & 0.1443 & -0.1336 & 0.2004 & 0.1443 & 0.3077 & 0.3649 & 0.2004 & 0.1066 & 0.1043 \\
\hline IPRSRs & $0.0381^{*}$ & 0.3702 & $0.0381^{*}$ & -0.2908 & -0.1762 & -0.2094 & -0.3409 & -0.1100 & 0.0529 & 0.1265 & 0.5568 \\
\hline IPRSRc & 0.2986 & 0.0843 & 0.2986 & -0.4479 & -0.2322 & -0.4778 & -0.1579 & 0.5954 & 0.4866 & -0.0970 & 0.5263 \\
\hline IPRSRdif & 0.2831 & 0.4360 & 0.2831 & -0.2735 & -0.1254 & -0.3570 & 0.0577 & 0.6846 & 0.4672 & 0.1818 & 0.1823 \\
\hline
\end{tabular}

* valor estatisticamente significante $p \geq 0,05$

Legenda: IPRSSs: índice percentual de reconhecimento de sentenças no silêncio sem as próteses auditivas. IPRSSc: índice percentual de reconhecimento de sentenças no silêncio com as próteses auditivas. IPRSRs: índice percentual de reconhecimento de sentenças no ruído sem as próteses auditivas. IPRSRc: índice percentual de reconhecimento de sentenças no ruído com as próteses auditivas. 
A adaptação da prótese auditiva é uma das condições fundamentais para que o deficiente auditivo desenvolva seu potencial, desde que ele utilize plenamente o aparelho, isto é, em todas as horas do dia ${ }^{22}$. A utilização do aparelho em mais de dois períodos por dia favorece a comunicação e promove o desenvolvimento de relacionamentos em deficientes auditivos ${ }^{23}$.

A mesma porcentagem $(46,2 \%)$ referiu fazer uso entre nove e 12 horas, ou mais de 12 horas diárias, perfazendo o uso superior a dois turnos, o que caracteriza o uso efetivo com os benefícios descritos pela literatura citada.

A perda auditiva tem um forte impacto sobre a vida psicossocial dos portadores dessa deficiência. A possibilidade de usar próteses auditivas, a fim de facilitar a comunicação e minimizar as dificuldades causadas pela perda auditiva, pode ser motivadora e significante para a melhoria da qualidade de vida dos mesmos ${ }^{24}$.

A consciência do paciente quanto à importância do aparelho em sua vida favorece não apenas o uso efetivo do mesmo, mas também a sua conservação e manutenção ${ }^{23}$.

É citado na literatura ${ }^{23}$, que no período da adolescência, o uso das próteses auditivas, embora necessário, torna-se uma grande preocupação para esses indivíduos. Ainda assim, existem adolescentes que não prescindem do uso das próteses auditivas, visto o benefício que obtêm com as mesmas.

Existe a possibilidade de ocorrer um aumento da intolerância para sons intensos do ambiente nos dois primeiros meses após a adaptação ${ }^{25}$ e existe uma redução desta intolerância após seis meses, quando ocorre a acomodação, ou seja, o usuário acostuma-se com essas situações ao longo do tempo em que faz uso da amplificação ${ }^{21}$. Assim, considerando que algumas avaliações do presente estudo foram realizadas com presença de ruído competitivo, é importante ressaltar que todos os pacientes faziam uso dos aparelhos auditivos há mais de dez meses quando avaliados.

Quando comparados os resultados obtidos por meio dos IPRSS e IPRSR, com e sem o uso de próteses auditivas, as medidas mostraram diferenças estatisticamente significantes, indicando que o desempenho com o uso das próteses auditivas, se comparado ao desempenho sem o uso das mesmas, foi melhor, assim como o desempenho no silêncio, se comparado ao desempenho no ruído.

Os resultados aqui encontrados, que evidenciaram melhora de todos os indivíduos frente aos estímulos de fala, tanto no silêncio como no ruído, são bastante satisfatórios, pois quando inicia-se um processo de seleção e adaptação de próteses auditivas é esperado que o indivíduo seja capaz de reconhecer, além de sons ambientais, os estímulos de fala, e com isso, seja capaz de estabelecer uma comunicação satisfatória em qualquer idade. No caso da população aqui analisada, isto é ainda mais importante, uma vez que foram avaliadas crianças e adolescentes que estão em fase escolar.

Assim sendo, quanto mais aproximados os limiares obtidos com próteses auditivas dos limiares de normalidade, maiores serão as chances deste indivíduo se comunicar de maneira eficiente. Porém, é importante salientar que obter apenas os limiares frente a sons sem significado não é uma garantia de que determinado aparelho está auxiliando a comunicação do paciente. Para isso é importante a aplicação de testes com sentenças que investiguem a capacidade de reconhecimento de fala em condições que simulem situações de comunicação.

Além disso, a obtenção de medidas que avaliem o reconhecimento de fala, tanto no silêncio como no ruído, é outro fator a ser salientado.

Foi verificado neste estudo que a melhora para reconhecer a fala no ruído não foi tão evidente quanto no silêncio, o que era esperado, uma vez que esta tarefa exige outras habilidades.

É citado na literatura ${ }^{26}$ que em ambiente ruidoso, ou situações adversas, o indivíduo pode apresentar inúmeras dificuldades na inteligibilidade da fala, pois o número de pistas cai significantemente, levando-os a utilizar somente pistas disponíveis na situação. Assim, ressalta-se a importância da realização de testes na presença de ruído, já que pacientes com as mesmas habilidades de reconhecimento de fala no silêncio podem apresentar resultados extremamente diferentes em ambientes ruidosos.

Escutar no ruído está relacionado com um alto grau de esforço ${ }^{27}$ e com uma baixa inteligibilidade da fala. Nestes ambientes, é necessário que os padrões de amplificação da prótese auditiva estejam bem ajustados para não causar desconforto auditivo, o que pode levar o usuário à desistência do uso da prótese nestas situações ${ }^{22}$.

Um estudo ${ }^{14}$ comparou o reconhecimento de fala no silêncio e no ruído por crianças com e sem dificuldades de aprendizagem e verificou melhora no desempenho dessas crianças no silêncio, quando comparado ao desempenho das mesmas no ruído.

Outro estudo ${ }^{28}$ comparou o desempenho em testes de fala, com e sem ruído competitivo, realizado com indivíduos jovens e com idosos, sendo que cada grupo era composto tanto por indivíduos com audição normal, como indivíduos usuários de AASIs com deficiência auditiva neurossensorial. Obtiveram como resultado, que a medida que o ruído é incrementado, o rendimento dos grupos 
diminui. Contudo o grupo de indivíduos com perda auditiva neurossensorial teve piores resultados no reconhecimento da mensagem. Constataram, ainda, que os usuários de próteses auditivas melhoraram seu rendimento fazendo uso das próteses, tanto no silêncio, quanto no ruído.

Em estudo ${ }^{15}$ analisando o reconhecimento de fala no silêncio e no ruído em escolares com e sem prática musical e de distintos níveis sócio-culturais, pôde ser constatado que, crianças com e sem prática musical de mesmo nível sócio-cultural, apresentaram desempenho semelhante no reconhecimento de sentenças no silêncio e no ruído, já as crianças com nível sócio-cultural baixo apresentaram desempenho pior que as crianças de nível sócio-cultural mais elevado, independentemente da prática musical, na tarefa de reconhecimento de sentenças no silêncio e no ruído.

Assim sendo, todos estes estudos mostram que sempre que a avaliação for realizada em situações que testem, além da audibilidade, as habilidades auditivas, como a de fala no ruído e de fechamento auditivo, de cada sujeito, e estes indivíduos tiverem qualquer variável complicadora, estes resultados poderão estar prejudicados, pois se trata de uma tarefa mais complexa. Aqui neste caso, isso se torna ainda mais evidente, pois trata-se de indivíduos que possuem perda auditiva, ou seja, o limiar de audibilidade está prejudicado, o que vai ter conseqüências importantes na evolução das habilidades, pois além do grau de comprometimento, tem que ser considerada a idade da detecção da deficiência e da intervenção.

O questionário de avaliação do beneficio em crianças e jovens ${ }^{21}$ forneceu informações importantes provenientes da própria criança, em relação ao benefício proporcionado pela amplificação sonora no comportamento auditivo das mesmas, em situações de vida diária.

Tais informações não poderiam ser obtidas por meio da bateria de testes padrão, uma vez que esses comportamentos tendem a ocorrer em casa e não em ambientes de testagem, os quais possuem controle do som ambiental ${ }^{29}$.

Por se tratar de um questionário com perguntas de fácil acesso e por possuir o auxílio de gravuras ilustrativas, o mesmo foi respondido sem dificuldades pela maioria dos pacientes.

Com relação ao benefício referido pelas 13 crianças, três delas referiram menores benefícios que as demais, o que pode estar relacionado ao tempo de uso diário dos AASIs, já que as mesmas referiram utilizá-los por menos de seis horas diárias, ou seja, menos de dois períodos diários. No entanto, essas mesmas crianças apresentaram benefício quando verificado através do teste de reconhecimento de fala, mostrando que a prótese está com bom ganho e proporciona benefício para os mesmos.

O uso não efetivo da prótese compromete a integração social, incluindo o meio educacional e ocupacional ${ }^{30}$. Assim, quanto maior a aceitação e o tempo de uso das próteses auditivas, o paciente torna-se mais independente, integrado à sociedade e melhora a sua qualidade de vida.

Sabe-se que o benefício das próteses auditivas está relacionado à melhora da comunicação na vida diária, incluindo a redução da inabilidade e da desvantagem auditiva ${ }^{31}$. Foi observado que o ambiente onde as crianças percebem maior benefício da protetização é o ambiente doméstico, onde as situações de escuta são mais ideais; em segundo lugar foi referido o ambiente escolar, onde a maior dificuldade encontrada foi para ouvir o professor quando o mesmo estava longe. Isso reforça a necessidade de se orientar quanto ao posicionamento do aluno próximo ao professor, pois este favorece a percepção do sinal de fala e reduz a interferência do ruído ambiental ${ }^{32}$.

$\mathrm{O}$ ambiente onde as crianças referiram menor benefício com o uso das próteses auditivas foi o ambiente social, principalmente para entender uma pessoa falando em ambiente com ruído intenso. Em ambientes ruidosos a fala é mascarada, dificultando assim a recepção da mensagem falada. Nesses ambientes, também é necessário que as próteses auditivas estejam bem reguladas para não causar desconforto auditivo, o que levaria o usuário a não utilizá-los nas situações de ruído ${ }^{21}$.

Após analisar individualmente os resultados das medidas objetiva e subjetiva de avaliação, os mesmos foram correlacionados, a fim de registrar o benefício proporcionado pelas próteses auditivas, levando em consideração as medidas quantitativas do LSP e a opinião dos pacientes.

Os resultados do IPRSS e IPRSR, com e sem próteses auditivas e a diferença entre os mesmos, foram correlacionados com a pontuação média entre todas as questões do questionário de Avaliação do Benefício do AASI em Crianças e Adolescentes (Tabela 4). Apesar de ter sido percebida melhora no desempenho por meio de ambas as avaliações, não foi constatada correlação estatisticamente significante entre os dados, o que indica que a melhora apresentada no teste LSP, tanto no silêncio, quanto no ruído, com e sem o uso das próteses auditivas, não corresponde proporcionalmente ao benefício percebido pelos pacientes por meio do questionário.

Como referido em um estudo ${ }^{33}$, os testes subjetivos têm como finalidade documentar as opiniões e atitudes do paciente, já os resultados objetivos utilizam procedimentos empíricos para verificar 
as melhoras no desempenho. Assim, o sucesso da adaptação de próteses auditivas depende da análise do benefício que a prótese proporciona para o usuário, e essas medidas são cada vez mais importantes em qualquer programa de adaptação de próteses auditivas ${ }^{34}$.

A utilização do questionário de avaliação do benefício em crianças e adolescentes ${ }^{21}$, com 0 propósito de avaliar o benefício fornecido pela amplificação sobre o ponto de vista do paciente, é um instrumento eficaz em fornecer informações sobre o benefício que a prótese auditiva proporciona em situações de rotina doméstica, escolar e social nas crianças e adolescentes avaliados.

Em um estudo realizado ${ }^{35}$ não foram encontradas diferenças entre os benefícios objetivo e subjetivo fornecidos pela amplificação sonora, tanto na avaliação após um mês, quanto na realizada após seis meses de uso de próteses auditivas

Outra pesquisa ${ }^{36}$ verificou a necessidade da utilização de protocolos de verificação e validação como parte dos procedimentos de indicação e adaptação de AASI na população infantil. Ainda, foi referido que os procedimentos de percepção da fala podem e devem ser realizados utilizando palavras familiares e com significado, uma vez que a familiaridade com as palavras pode interferir na melhora do desempenho nas avaliações.

Como mostra a Tabela 5 , os resultados do IPRSS e IPRSR, com e sem próteses auditivas e a diferença entre os mesmos foram, também, correlacionados com a pontuação de cada item do questionário. Não foi constatada correlação estatisticamente significante entre a maioria dos dados, o que indica que a melhora apresentada no teste IPRSS e IPRSR, com e sem o uso das próteses auditivas não corresponde proporcionalmente ao benefício percebido pelos pacientes por meio do questionário. Contudo, foi observada correlação estatisticamente significante do IPRSRs com as questões relacionadas ao ambiente doméstico, especificamente nas que questionam se o AASI ajuda a ouvir melhor a TV e se ajuda a ouvir melhor quando os pais chamam, além da correlação do IPRSSs com as questões referentes ao ambiente social, na questão sobre o AASI ajudar a brincar melhor com os amigos, o que mostra que os resultados são aleatórios, não apresentando uma relação fixa entre os mesmos.

A amplificação sonora por meio das próteses auditivas compensa a atenuação dos sons causada pela perda auditiva, mas muitas vezes, a distorção gerada por essa deficiência é mais difícil de ser compensada. Assim, as próteses auditivas melhoram a percepção da fala em condições de silêncio, devido a essa melhora na capacidade auditiva pela compensação da atenuação ${ }^{28}$, mas acredita-se que não há essa melhora no ruído por nem sempre conseguir reparar a distorção de forma satisfatória.

Um estudo realizado ${ }^{33}$ utilizou três testes com sentenças para o reconhecimento de fala com presença de ruído de fundo: o Revised Speech Perception in Noise test (R-SPIN), QuickSIN e Hearing in Noise Test (HINT). Estes foram comparados ao Hearing Aid Performance Inventory (HAPI), que consiste em um questionário para mensurar o desempenho na percepção da fala e o benefício advindo do uso da amplificação sonora. Nas análises realizadas, foi constatado que nos três testes de reconhecimento de fala, os pacientes apresentaram melhores resultados no silêncio e no ruído, quando em uso de próteses auditivas, se comparado aos resultados da avaliação sem as mesmas. As comparações entre os resultados subjetivos e objetivos documentados indicaram que à medida em a classificação no HAPI aumentava, o desempenho no R-SPIN, no HINT e no QuickSIN também melhorava. No entanto, correlações entre os resultados objetivos e subjetivos não foram significantes em todos os casos, o que sugere que a sensibilidade do HAPI e dos testes de reconhecimento de sentenças utilizados variam de acordo com itens e procedimentos específicos.

Acredita-se ainda, que estas divergências entre os testes objetivos e subjetivos são esperadas e bastante comuns, uma vez que os questionários levantam questões que estão relacionadas com expectativa, e essa é uma medida que tem inúmeras variáveis, as quais estão diretamente relacionadas com a forma que a audição ou a falta dela interfere na vida do indivíduo, aspectos emocionais e o quanto ele espera que a prótese deva suprir dificuldades que muitas vezes não estão diretamente ligadas a ela. Assim sendo, o paciente pode ter uma resposta ótima do ponto de vista do avaliador, mas considerá-la insuficiente, uma vez que ele o paciente - superestima o que a prótese deve oferecer de resultado. Por outro lado, o contrário também pode acontecer em pacientes menos exigentes e/ou mais realistas.

Assim sendo, esta pesquisa reforça a importância da utilização de instrumentos de medidas que permitam verificar, de forma objetiva, por meio de teste de reconhecimento de fala, e subjetiva, através de questionários, o resultado da amplificação sonora, principalmente em crianças e adolescentes, já que os testes com sentenças, quando aplicados no silêncio e no ruído, avaliam a comunicação do paciente em situações ideais e não ideais de escuta, enquanto o questionário permite conhecer como o paciente percebe o benefício proporcionado pela amplificação sonora em 
várias situações da vida diária, fazendo com que o audiologista tenha maior segurança com relação à protetização, tendo dados concretos do resultado da intervenção.

\section{CONCLUSÃO}

A partir dos IPRSS e IPRSR estabelecidos, nota-se melhor desempenho no reconhecimento das sentenças por parte das crianças e adolescentes nas situações em que fazem uso das próteses auditivas, tanto em ambientes silenciosos como ruidosos.
$\mathrm{Na}$ aplicação do questionário de avaliação do benefício, observou-se que em todos os itens avaliados a maioria das crianças e adolescentes referiu melhora no desempenho com o uso das próteses auditivas. Assim, foi verificado o benefício proporcionado pela amplificação sonora em diferentes situações da vida diária, considerando a opinião do paciente.

Ao comparar os resultados obtidos com o teste LSP e o questionário de auto-avaliação, não foi verificada correlação estatisticamente significante entre os mesmos, embora tenha havido bom desempenho em ambas as avaliações.

\begin{abstract}
Purpose: to evaluate children and teenagers musing hearing aids, investigate the benefit provided by sound amplification, through the Portuguese Sentences Lists Test and a benefit assessment questionnaire for children and teenagers; and to check the correlation between the results obtained from these two instruments. Method: 13 children and teenagers, between eight and 14 years old, both sexes, with a moderately severe hearing loss as the maximun level on the best ear, hearing aids users bilaterally for more than ten months and fitted by a Hearing Health Program were evaluated. An anamnesis was applied, then the subjects were submitted to a basic audiological evaluation, and were evaluated by the Portuguese Sentences Lists Test and a benefit assessment questionnaire for children and teenagers. Results: the results obtained from the Portuguese Sentences Lists Test and the questionnaire showed the subject's benefit with the use of the hearing aids, both in silence and in noise. However, there was no statistically significant correlation between these two instruments. Conclusion: although statistically significant correlation has not been verified between the results obtained using two assessment instruments, through the Portuguese Sentences Lists Test, the individuals had improvement in the performance of speech recognition, using the hearing aids, both in quiet and in noise, and in response to the questionnaire, the subjects reported benefit with the use of sound amplification.
\end{abstract}

KEYWORDS: Child; Hearing Loss; Hearing Aids; Speech Perception; Questionnaires 
Nome:

Idade:

Série:

Qual orelha usa AASI: ( ) OD ( ) OE ( ) Ambas

Uso do AASI:

( ) Somente em casa

( ) Somente na escola

( ) Somente na terapia de fono

( ) Em casa e na terapia

( ) En casa e na escola

( ) Em todos os lugares que vou

Eu uso o AASI:

( ) somente pela manhã

( ) somente pela tarde

( ) somente pela noite

( ) por dois períodos (

( ) O dia todo. Só tiro para tomar banho e dormir

Parte A: Em casa

1)O AASI faz com que eu ouça melhor a televisão?

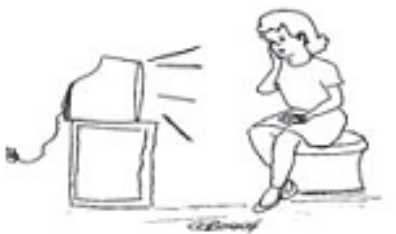
( ) $\operatorname{Sim}()^{-}$
( ) Não \&
( ) um pouco@

2) Com o AASI ouço melhor o telefone ou campainha?

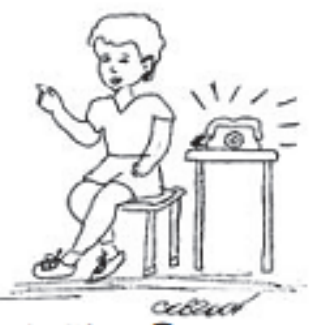

( ) $\operatorname{Sim}(\mathbf{C}$

( ) Não (8)

( ) um pouco $:)$

3) Com o AASI eu ouço meus pais me chamarem?

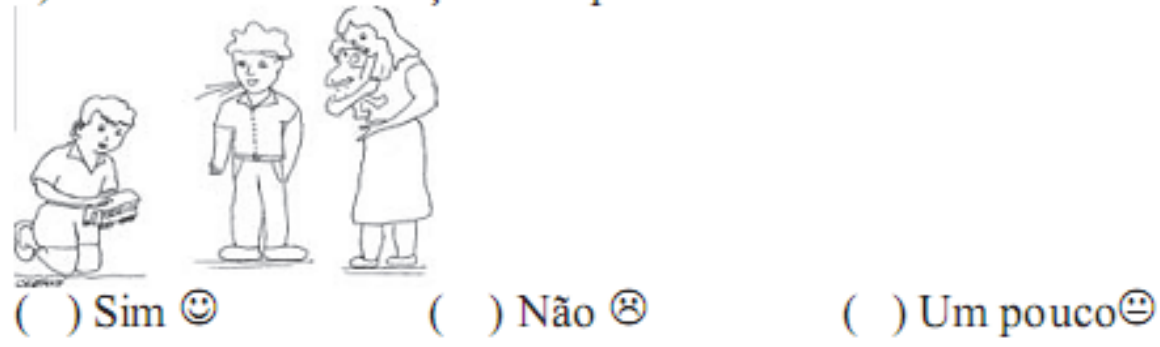




\section{Parte B: Na escola}

1) Com o AASI eu ouço o professor ?

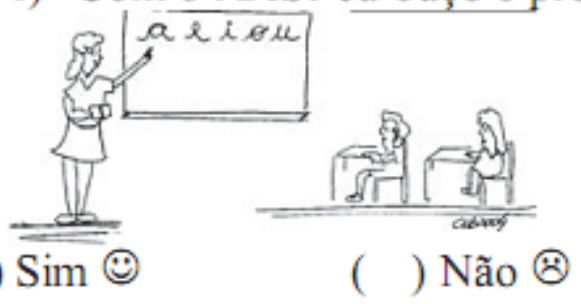

( ) Um pouco $:-$

2) Com o AASI eu ouço meus amigos de classe?

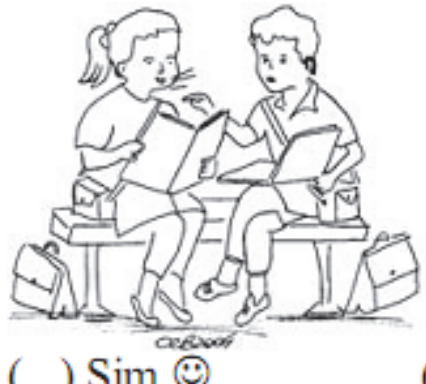
( ) $\operatorname{Sim}($ )
( ) Não • :
( ) Um pouco $:)$

3) Com o AASI eu ouço o professor quando ele está perto?

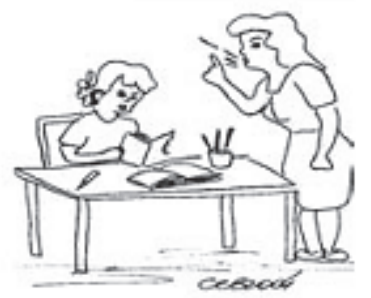
( ) $\operatorname{Sim}($ )
( ) Não *
( ) Um pouco $:-$

4) Com o AASI eu ouço o professor quando ele está longe?
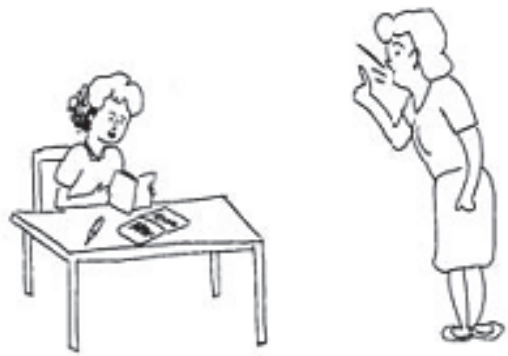

( ) $\operatorname{Sim}(\mathbf{0}$

( ) Não (*)

( ) Um pouco :- 


\section{Parte C: Social}

1) Com o AASI eu brinco melhor com meus amigos?

los.

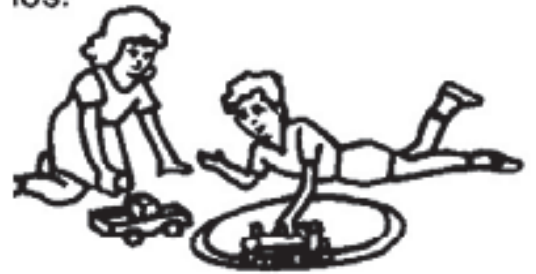
( ) $\operatorname{Sim}($ )
( ) Não (:)
( ) Um pouco $:-$

2) Com o AASI eu ouço meus amigos ?

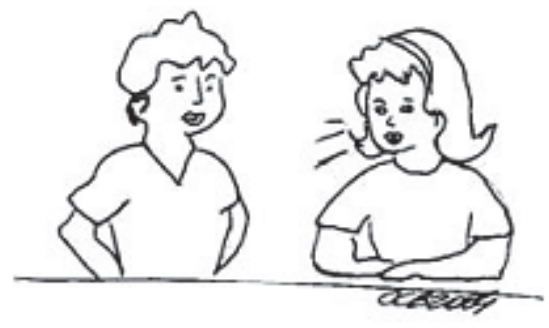
( ) $\operatorname{Sim}(;)$
( ) Não (*)
( ) Um pouco $:-$

3) Com o AASi eu ouço os carros passando na rua ?

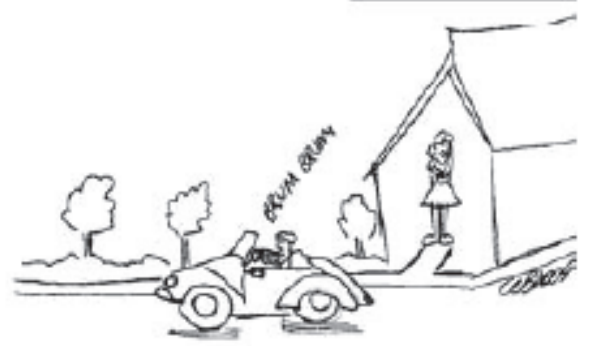
( ) $\operatorname{Sim}(-)$
( ) Não :
( ) Um pouco $\odot$

4) Com o AASI eu ouço uma pessoa num lugar com barulho?

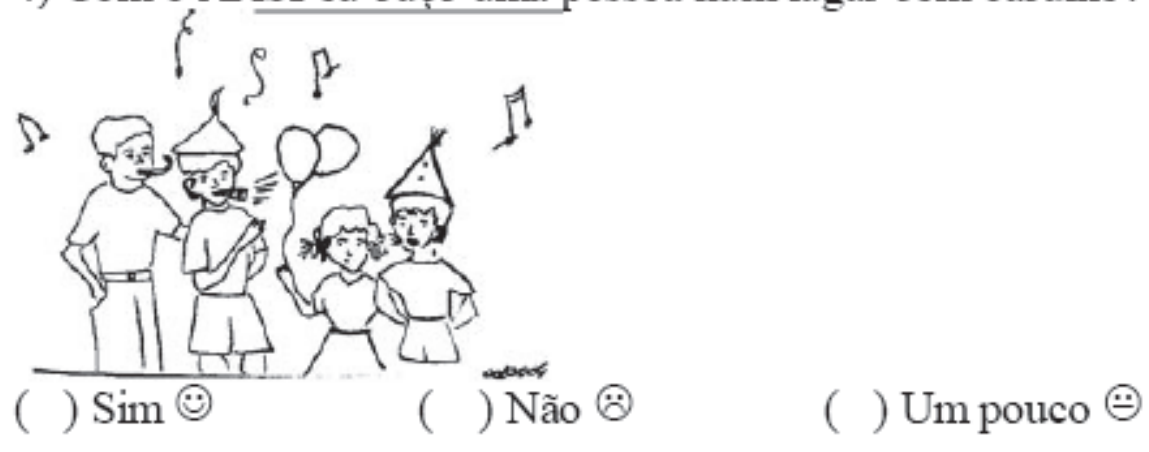

Figura 1 - Questionário de Avaliação do Benefício do AASI em Crianças e Jovens (Boscolo et al., 2006) 


\section{REFERÊNCIAS}

1. Pinotti KSA, Corazza MCA, Alcarás PAS. Avaliação Eletrofisiológica do Nervo Auditivo em Pacientes Normo-ouvintes com Ausência do Reflexo Estapediano. Arq. Int. Otorrinolaringol. 2009: 13(4): 386- 93.

2. Santos JN, Lemos SMA, Rates SPM, Lamounier JA. Habilidades auditivas e desenvolvimento de linguagem em crianças. Pró-Fono R. Atual. Cient. 2008; 20(4): 255-60.

3. Vieira ABC, Macedo LR, Gonçalves DUO. diagnóstico da perda auditiva na infância. Pediatria. 2007: 29(1): 43-9.

4. Campos AHC, Russo ICP, Almeida K. Indicação, seleção e adaptação de próteses auditivas. In: Almeida, K; lório, MCM. Próteses auditivas: fundamentos teóricos e aplicações clínicas. 2. ed. São Paulo: Lovise, 2003. p. 35-54.

5. Boéchat EM, Russo ICP, Almeida K. Reabilitação do adulto deficiente auditivo. In: Almeida K, lório MCM. Próteses auditivas: fundamentos teóricos e aplicações clínicas. 2. ed. São Paulo: Lovise, 2003. p. 437-46.

6. Couto MIV, Lichtig I. Efeitos da amplificação sonora sobre as modalidades comunicativas utilizadas pelos pais. Pró-Fono R. Atual. Cient. 2007: 19(1): 75-86.

7.PenMG, Magabeira-AlbernazPLDesenvolvimento de um teste para logoaudiometria: discriminação vocal. In: II Congresso Pan Americano de Otorrinolaringologia $Y$ Broncoesofasologia, LimaPeru, 1973. Anales, v.2, p 223-6.

8. Egan J. Articulation testing methods. Laryngoscope. 1948: 58: 955- 91.

9. Hirsh IJ, Davis H, Silverman SR, Reynolds FG, Eldert E, Benson RW. Development of materials for speech audiometry. J. Speech Hear. Disgord. 1952: 17: $321-37$

10. Matas CG, lorio MCM. Verificação e validação do processo de seleção e adaptação de próteses auditivas. In: Almeida, K; lório, MCM. Próteses auditivas: fundamentos teóricos e aplicações clínicas. 2. ed. São Paulo: Lovise, 2003. p. 305-23. 11. Kalikow DN, Stevens KN, Elliott LL. Development of a test intelligibility in noise using sentence materials with controlled word predictability. J. Acoust. Soc. Am. 1977: 61(1): 337-51.

12. Plop R, Mimpen AM. Improving the reability of testing the speech reception threshold for sentences. Audiology. 1979: 18:43-52.

13. Costa MJ. Listas de sentenças em português: apresentação \& estratégias de aplicação na audiologia. Santa Maria : Pallotti, 1998. p 44.

14. Daniel RC, Costa MJ, Oliveira TMT. Reconhecimento de sentenças no silêncio e no ruído de crianças com e sem histórico de repetência escolar. Fono atual. 2003: 26(4): 35-41.

15. Gambini C. Reconhecimento de fala em escolares com e sem prática musical e diferentes níveis sócioculturais [monografia de especialização]. Santa Maria: Universidade Federal de Santa Maria, Curso de Especialização em Fonoaudiologia, 2006.

16. Bess $\mathrm{FH}$, Hedley-Williams A, LLichtenstein MJ. Avaliação auditiva em idosos In.: Musiek, FE; Rintelmann, WF. Perspectivas atuais em avaliação auditiva. São Paulo: Monole: 2001, p-343-64.

17. Freitas CD, Costa MJ. Processo de adaptação de próteses auditivas em usuários atendidos em uma instituição pública federal - parte I: resultados e implicações com o uso da amplificação. Rev Bras Otorrinolaringol. 2007: 73(6): 744-51.

18. Lloyd LL, Kaplan H. Audiometric interpretation: a manual of basic audiometry. Baltimore: University Park Press, 1978.

19. Levitt $H$, Rabiner LR. Use of a sequencial strategy in intelligibility testing. J. Acoust. Soc. Am. 1967: 42: 609-12.

20. Boothroyd A. Speech perception, sensorioneural hearing loss and hearing Aids. In: Studebaker, G; Hochberg, I. Acoustical Factors Affecting Hearing Aid Performance. 2Ed. Boston: Allyn \& Bacon, 1993. p. 277-99.

21. Boscolo CC, Costa MPR, Domingos CMP, Perez FC. Avaliação dos benefícios proporcionados pelo AASI em crianças e jovens da faixa etária de 7 a 14 anos. Rev. Bras. Ed. Esp. 2006: 12(2): 255-68. 22. Menegotto IH, lorio MCM. Processamento dos Sinais sonoros nas Próteses Auditivas: Compressão. In: Almeida K, lório MCM. Próteses Auditivas: fundamentos teóricos e aplicações clínicas. 2. ed. São Paulo: Lovise, 2003. p. 119-48. 23. Zugliani AP, Motti TFG, Castanho RMO. Autoconceito do adolescente deficiente auditivo e sua relação com o uso do Aparelho de Amplificação Sonora Individual. Rev. Bras. Ed. Esp. 2007: 13(1): 95-110.

24. Gil D. Treinamento auditivo formal em adultos com deficiência auditiva. São Paulo, 2006, p. 181, (Tese de Doutorado - Universidade Federal de São Paulo).

25. Bucuvic EC, lório MCM. Benefícios e dificuldades auditivas: um estudo em novos usuários de prótese auditiva após dois e seis meses de uso Fono Atual. 2004: 29(7): 19-29.

26. Aurélio NHS, Becker KT, Padilha CB, Santos SN, Petry T, Costa MJ. Limiares de reconhecimento de sentenças no silêncio em campo livre versus limiares tonais em fone em indivíduos com perda auditiva coclear. Rev. CEFAC.2008; 10(3): 378-84. 27. Larsby B, Hällgren M, Lyxell B, Arlinger S. Cognitive performance and perceived effort in 
speech processing tasks: effects of different noise backgrounds in normals and in hearing-impaired subjects. Int J Audiol. 2005: 44: 131-43.

28. Hällgren M, Larsby $B$, Lyxell $B$, Arlinger $S$. Speech understanding in quiet and noise, with and without hearing aids. Int J Audiol. 2005: 44: 574-83. 29. Osberger MJ, Geier L, Zimmerman-Phillips S, Barker MJ. Use of a Parent-Report Scale to assess benefit in children given the Clarion Cochlear Implant. Am J Otol. 1997: 18: 79-80.

30. Lessa AH, Costa MJ, Becker KT, Vaucher AVA. Satisfação de Usuários de Próteses Auditivas com Perda Auditiva de Graus Severo e Profundo. Arq. Int. Otorrinolaringol. 2010; 14(3): 338-45.

31. Almeida K. Avaliação dos resultados da intervenção. In: Almeida K, lório MCM, organizadoras Próteses auditivas: fundamentos teóricos e aplicações clínicas. $2^{\underline{a}}$ ed. São Paulo: Lovise; 2003. p.335-53.

32. Marriage JE, Moore BCJ, Stone MA, Baer T. Effects of three amplification strategies on speech perception by children with severe and profound hearing loss. Ear Hear. 2005: 26(1): 35-47.

33. Mendel LL. Objective and Subjective Hearing Aid Assessment Outcomes. American Journal of Audiology. 2007:16 (2): 118-29.

34. Haskell GB. Noffsinger D, Larson VD, Williams DW, Dobie RA, Rogers JL. Subjective Measures of Hearing Aid Benefit in the NIDCD/VA Clinical Trial. Ear Hear. 2002: 23(4): 301-7.

35. Humes LE, Humes LE, Wilson DL. A comparison of single-channel linear amplification and twochannel wide-dynamic-range-compression amplification by means of an independent-group design. Am. J. Audiol. 2004: 13: 39-53.Larry E Humes, Lauren E Humes, Dana L Wilson

36. Rissatto AR, Novaes BCAC. Próteses auditivas em crianças: importância dos processos de verificação e validação. Pró-Fono R. de Atual. Cient. 2009: 21(2): 131-6.
http://dx.doi.org/10.1590/S1516-18462012005000058

RECEBIDO EM: 01/03/2011

ACEITO EM: 16/11/2011

Endereço para correspondência:

Enma Mariángel Ortiz Torres

Avenida Presidente Vargas, 1720, 302

Santa Maria - RS

CEP: 97015-510

E-mail: emmita_06@hotmail.com 